\title{
Baicalin Attenuates Cardiac Dysfunction and Myocardial Remodeling in a Chronic Pressure-Overload Mice Model
}

\author{
Yanqing Zhanga Pingping Liao ${ }^{a} \quad$ Meng'en Zhu ${ }^{a}$ Wei Lia Dan Hu ${ }^{b}$ Long Chen \\ Siming Guan ${ }^{a}$
}

aDepartment of Geriatrics, Union Hospital, Tongji Medical College, Huazhong University of Science and Technology, Wuhan, bDepartment of Cardiovascular Surgery, Union Hospital, Tongji Medical College, Huazhong University of Science and Technology, Wuhan, 'Clinical Center for Human Genomic Research, Union Hospital, Huazhong University of Science and Technology, Wuhan, China

\section{Key Words}

Hypertrophy • Pressure overload • Baicalin

\begin{abstract}
Background/Aims: Baicalin has been shown to be effective for various animal models of cardiovascular diseases, such as pulmonary hypertension, atherosclerosis and myocardial ischaemic injury. However, whether baicalin plays a role in cardiac hypertrophy remains unknown. Here we investigated the protective effects of baicalin on cardiac hypertrophy induced by pressure overload and explored the potential mechanisms involved. Methods: C57BL/6J-mice were treated with baicalin or vehicle following transverse aortic constriction or Sham surgery for up to 8 weeks, and at different time points, cardiac function and heart size measurement and histological and biochemical examination were performed. Results: Mice under pressure overload exhibited cardiac dysfunction, high mortality, myocardial hypertrophy, increased apoptosis and fibrosis markers, and suppressed cardiac expression of PPAR $\alpha$ and PPAR $\beta / \delta$. However, oral administration of baicalin improved cardiac dysfunction, decreased mortality, and attenuated histological and biochemical changes described above. These protective effects of baicalin were associated with reduced heart and cardiomyocyte size, lower fetal genes expression, attenuated cardiac fibrosis, lower expression of profibrotic markers, and decreased apoptosis signals in heart tissue. Moreover, we found that baicalin induced PPAR $\alpha$ and PPAR $\beta / \delta$ expression in vivo and in vitro. Subsequent experiments demonstrated that long-term baicalin treatment presented no obvious cardiac lipotoxicity. Conclusions: The present results demonstrated that baicalin attenuates pressure overload induced cardiac dysfunction and ventricular remodeling, which would be due to suppressed cardiac hypertrophy, fibrosis, apoptosis and metabolic abnormality.
\end{abstract}

Y. Zhang and P. Liao contributed equally to this work. 


\section{Cellular Physiology Cell Physiol Biochem 2017;41:849-864 \begin{tabular}{ll|l} 
and Biochemistry Published onIIne: February 16, 2017 & $\begin{array}{l}\text { (c) } 2017 \text { The Author(s). Published by S. Karger AG, Basel } \\
\text { www.karger.com/cpb }\end{array}$ \\
\hline
\end{tabular} \\ Zhang et al.: Baicalin Attenuates Cardiac Hypertrophy}

\section{Introduction}

During the past half-century, great advances in the prevention, diagnosis, and treatment of cardiovascular diseases have been made with the notable exception of heart failure, which is a major global health problem with an estimated prevalence of 38 million patients worldwide [1]. An important cause of heart failure is chronic pressure overload due to aortic stenosis or hypertension, which leads to cardiac hypertrophy that may progress to heart failure. Uncompensated cardiac hypertrophy is characterized by enhanced expression of fetal genes, excessive increase in myocardial mass, abnormal accumulation of extracellular matrix, and severe abnormality of energy metabolism [2-4]. Growing evidence has confirmed that uncompensated cardiac hypertrophy is correlated with an increase in glucose utilization and a decrease in fatty acid utilization (FAU). This metabolic substrate switch seems to involve changes in mRNA level of genes implicated in the transport and metabolism of glucose and fatty acids, which are primarily regulated by a class of nuclear receptors called peroxisome proliferator-activated receptors (PPARs) [3].

PPAR $\alpha$ is a key regulator of myocardial fatty acid uptake and oxidation in the heart [5]. During pathological growth of heart failure, down-regulation of FAU genes correlates with decreased PPAR $\alpha$ level [6]. It has been shown that activation of PPAR $\alpha$ with mediumchain triglycerides is able to prevent the reduction of FAU and reverse the development of cardiac hypertrophy $[6,7]$. However, mice with cardiac-restricted overexpression of PPAR $\alpha$ (MHC-PPAR $\alpha$ ) has been shown to develop cardiomyopathy with cardiac dysfunction, and enhanced myocardial lipid accumulation followed by high fat diet (HFD) [5, 8]. Similar to PPAR $\alpha, \operatorname{PPAR} \beta / \delta$ is also highly expressed in cardiac myocytes and necessary for maintaining myocardial lipid homeostasis [9]. Cardiac PPAR $\beta / \delta$ level was repressed by pressure overload in a hypoxia dependent pathway [10]. Mice with cardiac-specific deletion of the PPAR $\beta / \delta$ has been shown to develop cardiomyopathy with cardiac dysfunction and myocardial lipid accumulation $[10,11]$. Conversely, mice with cardiac-restricted overexpression of PPAR $\beta / \delta$ (MHC-PPAR $\beta / \delta$ ) did not accumulate myocardial lipid, and exhibited increased myocardial glucose utilization and normal cardiac function [8]. Additionally, reactivation of cardiac $\operatorname{PPAR} \beta / \delta$ is sufficient to reverse cardiac hypertrophy in vitro and in vivo $[10,12]$.

Baicalin (Fig. 1) is is a natural flavone compound abundant in the rhizome of the perennial herb Scutellaria baicalensis, known as huangqin in Chinese traditional medicine [13]. By using $\mathrm{ApoE}^{-/-}$mice, our group previously demonstrated that baicalin attenuates atherosclerosis through lipids regulation and dendritic cells inhibition [14, 15]. Another evidence revealed protective effects of baicalin on ER stress-induced cardiomyocytes apoptosis, acute myocardial infarction injury, and hypoxic pulmonary hypertension [16-18]. However, no study has addressed the effect of baicalin on pressure overload-induced cardiac hypertrophy. Therefore, in this study, we aimed to investigate the potential role of baicalin on pressure overload induced heart failure.

\section{Materials and Methods}

\section{Ethics statement}

All animal experiments were performed in accordance with the Guide for the Care and Use of Laboratory Animals published by the NIH and approved by The Institutional Animal Care and Use Committee at Tongji Medical College, Huazhong University of Science and Technology (IACUC Number: 477).

Animals and experimental groups

Male C57BL/6 mice aged 8-9 weeks were purchased from Beijing HFK Bioscience (Beijing, China). All animals were kept on a chow diet in a 12-hour light/12-hour dark environment at $25^{\circ} \mathrm{C}$ in the Tongji Medical School Experimental Animal Center. Mice were randomly assigned to four groups: Sham with vehicle (Sham+Veh), Sham with baicalin (Sham+BAI), TAC with vehicle (TAC+Veh), TAC with baicalin (TAC+BAI). Mice were treated orally with baicalin (gavage, $100 \mathrm{mg} / \mathrm{kg} /$ day) or vehicle for 4 weeks following TAC or 


\section{Cellular Physiology Cell Physiol Biochem 2017;41:849-864

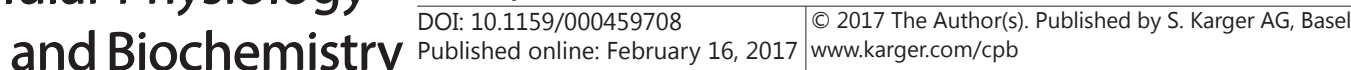

Sham operation. In some experiments, mice were treated orally with baicalin or vehicle for 8 weeks following TAC operation. For diet studies, mice were allowed ad libitum access to HFD for 4 weeks, which provides $43 \%$ of the calories from fat (Beijing HFK Bioscience, Beijing, China), accompanied by daily administration (gavage, $100 \mathrm{mg} / \mathrm{kg} /$ day) of baicalin or vehicle. At the end of each experiment, mice were sacrificed by cervical vertebra dislocation following anesthesia with pentobarbital sodium $(50 \mathrm{mg} / \mathrm{kg}$ )

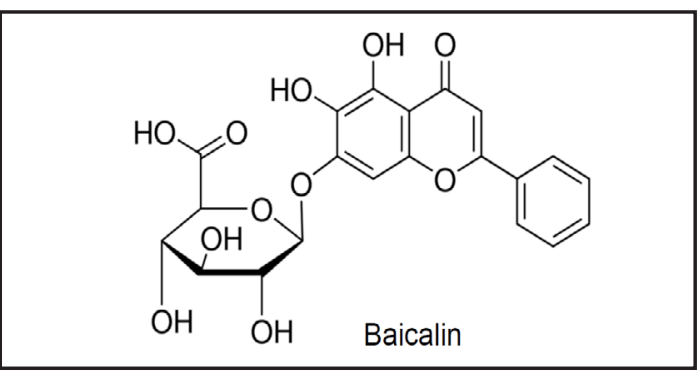

Fig. 1. The chemical structures of baicalin. intraperitoneally. Then, hearts were harvested, weighed, excised and snap-frozen in liquid nitrogen for protein and RNA extraction or fixed for histological analysis. In the present study, baicalin was purchased from Sigma (St. Louis, Missouri, USA) and dissolved in normal saline and administrated by gavage. The concentration of baicalin $(100 \mathrm{mg} / \mathrm{kg} /$ day) were chosen according to our previous studies $[14,15]$.

\section{Transverse aortic constriction (TAC)}

We established pressure overload-induced cardiac hypertrophy via TAC as previously described [19]. Briefly, mice were anaesthetized with $3 \%$ pentobarbital sodium $(50 \mathrm{mg} / \mathrm{kg})$. A thoracotomy was performed to visualize the aortic arch. TAC was created using a 6-0 suture banded between the carotid arteries over a 27-gauge needle. The needle was then gently removed, creating a 60-80\% constriction with an aortic outer diameter of approximately $0.3 \mathrm{~mm}$. Sham control animals underwent corresponding surgery without aortic restriction. Following chest closing and skin suturing, mice were allowed to recover and continued with the experimental process.

\section{Echocardiography}

We used a Vevo 2100 high-resolution microimaging system equipped with a $30-\mathrm{MHz}$ transducer (VisualSonics, Toronto, Canada) for echocardiography. Mice were anesthetized with 1.5\% isoflurane and two-dimensional echocardiographic views of the parasternal long axes and the mid-ventricular short axis were obtained. We measured the left ventricular end-diastolic diameter (LVEDD), and left ventricular end-systolic diameter (LVESD), interventricular septal thickness in diastole (IVSd) and in systole (IVSs) in M-mode. Left ventricular fractional shortening (FS) and ejection fraction (EF) were calculated as previously described [20]. All of these parameters were obtained from at least three beats and averaged subsequently.

\section{Histology and TUNEL assay}

Fixed heart tissues were embedded in paraffin and cut into $5-\mu$ m thickness sections, followed by staining with hematoxylin and eosin (H\&E) or Masson's trichrome. Frozen sections were prepared from frozen heart tissue embedded with optimum cutting temperature (OCT) media and stained with Oil Red O. For Terminal deoxynucleotidyl transferase mediated dUTP nick end labeling (TUNEL) assay, myocardial sections were treated as instructed in the protocol of In Situ Cell Death Detection kit (Roche Diagnostics GmbH, Mannheim, Germany). Digital images were obtained with a $40 \times$ objective lens by microscopy (Olympus, Tokyo, Japan). H\&E-stained transverse sections were chosen to measure single cardiomyocytes cross-sectional area. We selected and counted more than 100 myocytes to assess the mean cross-sectional area using Image-Pro Plus (Media Cybernetics, Houston, USA). For the measurement of fibrosis, we randomly selected 30 fields from Masson trichrome-stained sections and analyzed the degree of fibrosis using Image-Pro Plus. We counted the ratio of TUNEL-positive nuclei and total nuclei per $40 \times$ objective field to determine the percentage of apoptotic cells. More than 5 fields per animal were examined in a blinded fashion by a technician who was not informed about the experiment groups.

\section{Biochemical analysis}

Blood samples were collected into tubes and centrifuged for serum separation. Serum non-esterified fatty acids (NEFA) levels were measured using a commercially available reagent according to manufacturer's guideline. Measurement of heart lipid content was performed as previously described [21]. Briefly, $50 \mathrm{mg}$ of heart tissue was weighed and homogenized with $1 \mathrm{ml}$ of buffer containing $18 \mathrm{mM}$ Tris, pH 7.5, $300 \mathrm{mM}$ mannitol, $50 \mathrm{mM}$ EGTA, and $0.1 \mathrm{mM}$ phenylmethysulfonyl fluoride. $400 \mu \mathrm{l}$ of above homogenate was mixed 


\section{Cellular Physiology Cell Physiol Biochem 2017;41:849-864

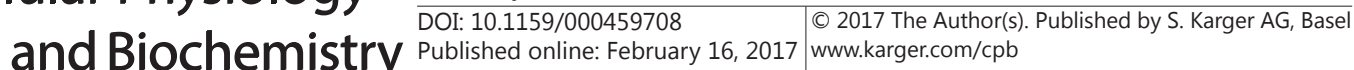 \\ Zhang et al.: Baicalin Attenuates Cardiac Hypertrophy}

with $4 \mathrm{ml}$ of chloroform/methanol (2:1) and incubated at room temperature with continuous shaking overnight. Then $800 \mu \mathrm{l}$ of $\mathrm{H}_{2} \mathrm{O}$ was added, vortexed, and centrifuged at 3,000g for $5 \mathrm{~min}$, and then the lower lipid phase was collected and dried under nitrogen gas. The lipid pellets were dissolved with a mixture of 60 $\mu \mathrm{l}$ of tert-butyl alcohol and $40 \mu \mathrm{l}$ of Triton X-114/methanol (2:1) mixture. The concentrations of triglyceride were then measured using the Stanbio assay kits.

\section{Cell culture and treatment}

H9C2 cell line were purchased from the Shanghai Cell Institute Country Cell Bank. Cells were cultured in low-glucose Dulbecco Modified Eagle Medium (DMEM, Gibco, USA) supplemented with $10 \%$ fetal bovine serum (FBS, Hyclone, USA), $100 \mathrm{mg} / \mathrm{ml}$ streptomycin and $100 \mathrm{U} / \mathrm{ml}$ penicillin (Hyclone, USA), under a $5.0 \%$ $\mathrm{CO}_{2}$ atmosphere, at $37^{\circ} \mathrm{C}$. After 48 hours, the culture medium was replaced with DMEM containing baicalin or dimethylsulfoxide (DMSO, Sigma, USA). After 24 hours of pretreatment, the culture medium was replaced with DMEM containing four different compositions: DMSO, baicalin, phenylephrin (PE, Cayman Chemical, USA), and baicalin plus PE. Following 48 hours of incubation, the cells were washed with cold Phosphate Buffer Solution (PBS) and fixed for immunofluorescent assay, or collected for Real time polymerase chain reaction (RT-PCR) or Western Blot assay. In the above experiment, baicalin and PE was dissolved in DMSO. The concentrations of baicalin $(50 \mu \mathrm{M})$ or PE $(100 \mu \mathrm{M})$ were chosen according to previous studies $[17,22]$.

\section{Immunofluorescent assay}

Cell contour was analyzed by immunofluorescence staining of $\alpha$-actinin protein in cultured H9C2 cells. Briefly, cultured cells were washed three times in PBS before being fixed in $4 \%$ paraformaldehyde for 15 minutes, then washed three times in PBS before being ruptured with $0.1 \%$ Triton-100. Slides were stained for cells shapes with $\alpha$-actinin antibody and for nuclei with DAPI. Digital images were obtained with a $40 \times$ lens by microscopy. Cell surface was measured by Image-Pro Plus. More than 60 random cells from three dependent experiments were selected for measurement and analyses of cell surface area.

\section{Gene expression analyses}

Total RNA was extracted from heart tissue or cultured cells with Trizol (Takara Biotechnology, Japan) and reverse-transcribed into CDNA, according to the manufacturer's protocol. Polymerase chain reactions were performed in an ABI PRISM 7900 Sequence Detector system (Applied Biosystems, Foster City, USA) using SYBR green as the fluorescence dye. The mRNA expression level of the target genes was normalized to the control $\beta$-actin using the comparative threshold cycle $\left(2^{-\Delta \Delta t}\right)$ method. The sequences of primers for RT-PCR were listed in Table 1.

\section{Western Blot}

Total protein from heart tissues or cultured cells was extracted using radioimmunoprecipitation lysis buffer (Beyotime, Shanghai, China). After denaturation and SDS-PAGE electrophoresis, separated proteins were transferred to nitrocellulose membranes. The primary antibodies used were as follows: anti-ANP (1:1000, Sigma, St. Louis, Missouri, USA); anti-PPAR $\alpha$ (1:1000, Santa Cruz Biotechnology, USA); anti-PPAR $\beta / \delta$ (1:1000, Santa Cruz Biotechnology, USA); anti-Bcl2 (1:1000, Proteintech, Wuhan, China); anti-Bax (1:1000, Proteintech, Wuhan, China); and anti-GAPDH (1:1000; Abcam, Cambridge, USA). Membranes were blocked in Tris Buffered Saline with Tween (TBST) containing 5\% skim milk and then incubated with primary antibodies at $4^{\circ} \mathrm{C}$ overnight. After 3 times of washing with TBST, the membranes were further incubated with HRP-conjugated secondary antibodies at room temperature for 2 hours. Bands were visualized with chemiluminescence assays (ECL detection reagents, Pierce, USA) and quantified by densitometry using BioRad's Quantity One software (version 4.4).

\section{Statistical analysis}

Data are presented as the mean \pm SEM. Differences between groups were compared by one-way ANOVA. We used the Kaplan-Meier method for survival analysis; we tested between-group differences in survival using the log-rank (Mantel-Cox) test. All statistical analyses were performed using Prism 5 (GraphPad Software Inc., San Diego, USA). $P<0.05$ was considered statistically significant. 


\section{Cellular Physiology Cell Physiol Biochem 2017;41:849-864

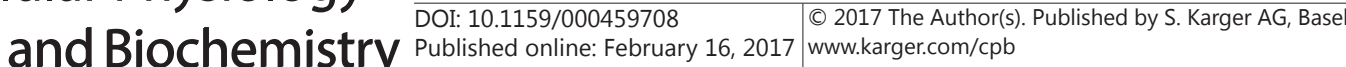 \\ Zhang et al.: Baicalin Attenuates Cardiac Hypertrophy}

Table 1. primers used for RT-PCR. Abbreviations: $r$, rat; $m$, mouse; $A N P$, natriuretic peptide type $\mathrm{A}$; $B N P$, natriuretic peptide type $\mathrm{B}$; $\beta$-MHC, myosin, heavy polypeptide 7; Col1 $\alpha 1$, collagen, type I, alpha 1; Col3 $\alpha 1$, collagen, type III, alpha 1 ; CTGF, connective tissue growth factor; Hk1, hexokinase 1; Glut1, Glucose transporter 1; Glut4, Glucose

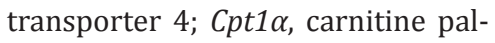
mitoyltransferase $1 \alpha$; Mcad, medium-chain acyl-CoA dehydrogenase; Lcad, long-chain acyl-CoA dehydrogenase; Dgat2, diacylglycerol 0-acyltransferase 2; Ppara, peroxisome proliferator activated receptor alpha; $\operatorname{Ppar} \beta / \delta$, peroxisome proliferator activated receptor beta, delta

\begin{tabular}{|c|c|c|}
\hline Genes & Forward & Reverse \\
\hline$r-A N P$ & 5'-AGGCCATATTGGAGCAAATC-3' & 5'-CATCTTCTCCTCCAGGTGGT-3' \\
\hline$r-B N P$ & $5^{\prime}$-TCAAAGGACCAAGGCCCTAC- $3^{\prime}$ & 5'-TAAAACAACCTCAGCCCGTCA-3' \\
\hline$r-\beta-M H C$ & 5'-AACCTGTCCAAGTTCCGCAAGGTG-3' & 5'-CAGCTGGGTAGCACAAGAGCTATC-3' \\
\hline$r-\beta$-actin & 5'-AGATCAAGATCATTGCTCCTCCT-3' & $5^{\prime}$-ACGCAGCTCAGTAACAGTCC- $3^{\prime}$ \\
\hline$m-A N P$ & $5^{\prime}$-TGACAGGATTGGAGCCCAGAG-3' & 5'-AGCTGCGTGACACACCACAAG-3' \\
\hline$m-B N P$ & 5'-ATCGGATCCGTCAGTCGTTTG-3' & 5'-CCAGGCAGAGTCAGAAACTGGAG-3' \\
\hline$m-\beta-M H C$ & $5^{\prime}$-ACAGGAAGAACCTACTGCGG-3' & $5^{\prime}$-CTCATTCAGGCCCTTGGCAC-3' \\
\hline$m-\operatorname{Col} 1 \alpha 1$ & 5'-ACGTGGAAACCCGAGGTATG-3' & 5'-CTTGGGTCCCTCGACTCCTA-3' \\
\hline$m-\operatorname{Col} 3 \alpha 1$ & 5'-GAGGAATGGGTGGCTATCCG-3' & 5'-TGGGCCTTTGATACCTGGAG-3' \\
\hline$m-C T G F$ & 5'-GTGTGCACTGCCAAAGATGG-3' & 5'-TGGGCCAAATGTGTCTTCCA- $3^{\prime}$ \\
\hline$m-H k 1$ & 5'-AGTGGAAGCCAGCTTTTTGA-3' & 5'-TTCAGCAGCTTGACCACATC-3' \\
\hline m-Glut1 & $5^{\prime}$-СCTGTCTCTTCCTACCCAACC-3' & 5'-GCAGGAGTGTCCGTGTCTTC-3' \\
\hline$m$-Glut4 & 5'-CTCATGGGCCTAGCCAATG-3' & 5'-GGGCGATTTCTCCCACATAC-3' \\
\hline$m-C d 36$ & 5'-GAGCCTTCACTGTCTGTTGGAA-3' & $5^{\prime}$-CTGCTACAGCCAGATTCAGAACTG- $3^{\prime}$ \\
\hline$m-C p t 1 \alpha$ & 5'-AGGACCCTGAGGCATCTATT-3' & 5'-ATGACCTCCTGGCATTCTCC-3' \\
\hline$m-M c a d$ & 5'-TGACGGAGCAGCCAATGA-3' & 5'-TCGTCACCCTTCTTCTCTGCTT-3' \\
\hline$m-L c a d$ & 5'-TTACATGCTGAGTGCCAACATG-3' & 5'-CGCCTCCGAGCAAAAGATT-3' \\
\hline$m$-Dgat2 & 5'-GTGGCCTGCAGTGTCATCCT-3' & 5'-TGGGCGTGTTCCAGTCAAAT-3' \\
\hline m-Ppara & 5'-ATGCCAGTACTGCCGTTTTC- $3^{\prime}$ & 5'-GGCCTTGACCTTGTTCATGT-3' \\
\hline m-Ppary & 5'-AAGAGCTGACCCAATGGTTG-3' & 5'-ACCCTTGCATCCTTCACAAG-3' \\
\hline$m-P p a r \beta / \delta$ & 5'-TGGAGCTCGATGACAGTGAC-3' & 5'-GTACTGGCTGTCAGGGTGGT-3' \\
\hline$m$ - $\beta$-actin & $5^{\prime}$-AAGGCCAACCGTGAAAAGAT- $3^{\prime}$ & 5'-GTGGTACGACCAGAGGCATAC-3' \\
\hline
\end{tabular}

Table 2. Parameters in mice at 4 weeks after sham operation or TAC. Data are mean \pm SEM. ${ }^{*} P<0.05$ vs. Sham + Veh; ${ }^{*} P<0.05$ vs. TAC + Veh. Abbreviations: $B W$, body weight; $H R$, heart rate; $L V E D d$, left ventricular end-diastolic diameter; LVESd, left ventricular end-systolic diameter; IVSd, interventricular septal thickness, diastolic; IVSs, interventricular septal thickness, systolic; $L V F S$, left ventricular fractional shortening; $L V E F$, left ventricular ejection fraction

\begin{tabular}{lllll}
\hline Parameters & $\begin{array}{l}\text { Sham + Veh } \\
(\mathrm{n}=8)\end{array}$ & $\begin{array}{l}\text { Sham }+ \text { BAI } \\
(\mathrm{n}=8)\end{array}$ & $\begin{array}{l}\text { TAC + Veh } \\
(\mathrm{n}=8)\end{array}$ & $\begin{array}{l}\text { TAC + BAI } \\
(\mathrm{n}=7)\end{array}$ \\
\hline$B W(\mathrm{~g})$ & $24.25 \pm 0.79$ & $23.65 \pm 1.81$ & $23.14 \pm 1.77$ & $24.12 \pm 2.32$ \\
HR (beats/min) & $493.65 \pm 60.24$ & $499.03 \pm 44.98$ & $481.58 \pm 39.13$ & $464.45 \pm 45.89$ \\
$\operatorname{LVEDd}(\mathrm{mm})$ & $3.04 \pm 0.34$ & $3.02 \pm 0.27$ & $4.26 \pm 0.33^{*}$ & $3.97 \pm 0.18$ \\
$\operatorname{LVESd}(\mathrm{mm})$ & $1.42 \pm 0.34$ & $1.36 \pm 0.19$ & $3.60 \pm 0.40^{*}$ & $2.99 \pm 0.31^{\#}$ \\
$\operatorname{IVSd}(\mathrm{mm})$ & $0.96 \pm 0.08$ & $0.95 \pm 0.09$ & $1.46 \pm 0.14^{*}$ & $1.20 \pm 0.14^{\#}$ \\
$\operatorname{IVSs}(\mathrm{mm})$ & $1.40 \pm 0.08$ & $1.44 \pm 0.10$ & $1.74 \pm 0.14^{*}$ & $1.55 \pm 0.12^{\#}$ \\
$\operatorname{LVFS}(\%)$ & $52.93 \pm 7.91$ & $54.21 \pm 5.56$ & $15.74 \pm 3.26^{*}$ & $23.68 \pm 5.21^{\#}$ \\
$\operatorname{LVEF}(\%)$ & $84.30 \pm 6.48$ & $83.51 \pm 5.13$ & $33.36 \pm 6.23^{*}$ & $47.37 \pm 8.97^{\#}$ \\
\hline
\end{tabular}

\section{Results}

Baicalin improves cardiac function and reduces mortality following TAC

To investigate the effect of baicalin on pressure overload-induced cardiac dysfunction, adult mice were randomly assigned to 4 groups: treatment with either baicalin or vehicle for 
4 weeks following either TAC or Sham operation. Then, left ventricular ejection fraction (EF), left ventricular fractional shortening (FS), interventricular septal thickness in diastole (IVSd) and in systole (IVSs), left ventricular end-diastolic diameter (LVEDD), and left ventricular end-systolic diameter (LVESD) were measured by echocardiography. Following 4 weeks of pressure overload, TAC mice displayed depressed cardiac function, increased left ventricular wall thickness, and increased left ventricular diameter (Table 2). However, we found that baicalin treatment resulted in attenuation of ventricular dysfunction, as evidenced by improvements in EF and FS (Table 2). Moreover, baicalin attenuated the increases of IVSd, IVSs, LVEDD, and LVESD induced by pressure overload (Table 2). No significant changes were observed in sham mice treated with baicalin or vehicle. Furthermore, we specially followed contractile functions and left ventricular diameters of TAC mice treated with baicalin or vehicle for up to 8 weeks. As shown in Fig. 2, baicalin treatment ameliorated adverse contractile dysfunction following TAC surgery, as measured by improvements in EF and FS (Fig. 2A and 2B). Additionally, baicalin attenuated left ventricular diameters dilation following TAC surgery, as assessed by decreases in LVESD and LVEDD (Fig. 2C, 2D and 2E). In a long-term animal experiment, we treated mice daily with baicalin or vehicle following TAC surgery. At the end of the 14 weeks, mortality in the baicalin-treated group was lower than that in the TAC group (Fig. 2F). Therefore, we concluded that baicalin treatment improved cardiac dysfunction and decreased mortality following TAC.

\section{Baicalin prevents pressure overload-induced cardiac hypertrophy}

In order to further evaluate the effects of baicalin on cardiac hypertrophy, we compared heart morphology and weight following TAC. As shown in Fig. 3, mice under pressure overload displayed marked elevation of heart weight/body weight (HW/BW) ratio (Fig. 3A), heart weight/ tibia length (HW/TL) ratio (Fig. 3B), and cardiomyocyte cross-sectional area (Fig. 3D and 3E). However, baicalin treatment resulted in attenuation of cardiac hypertrophy,

Fig. 2. Baicalin improves cardiac function and inhibits cardiac pathologic dilatation following TAC. Mice were anesthetized and performed transthoracic echocardiography at different time points: before TAC surgery (baseline), 2 , 4, 6 and 8 weeks after TAC surgery. Evaluation of (A) LVEF, (B) LVFS, (C) LVEDd, and (D) LVESd were shown in the indicated images. ${ }^{*} P$ $<0.05$ vs. TAC+Veh group at corresponding time point. ( $\mathrm{n}=8$ ) (E) Representative M-mode echocardiography images of the left ventricular. (F) Kaplan-Meier survival curves for different groups $(\mathrm{n}=18)$.

\section{A}

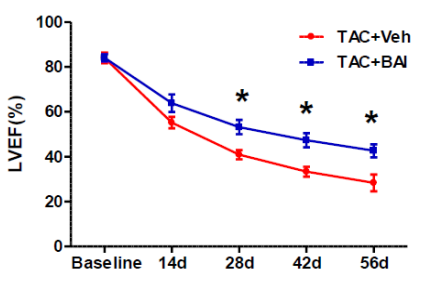

c

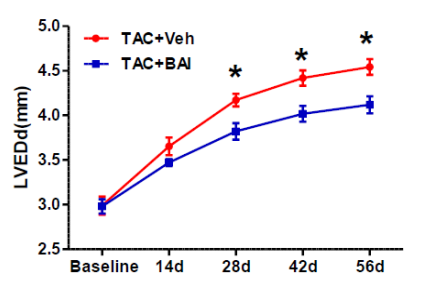

$E$

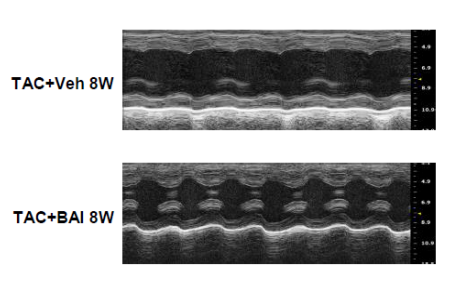

B

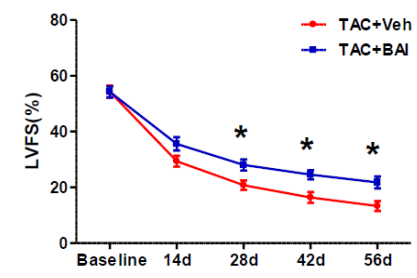

D

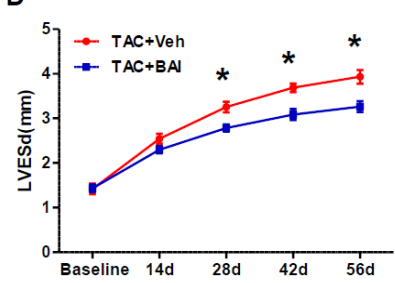

$\mathrm{F}$

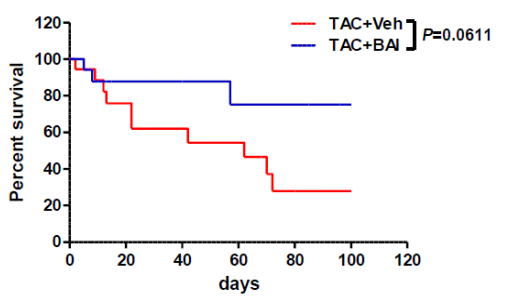


Fig. 3. Treatment with baicalin blunts cardiac hypertrophy in vivo. (A, $B$ and C) Statistical results of HW/BW, HW/ $\mathrm{TL}$, and $\mathrm{LW} / \mathrm{BW}$ for up to 4 weeks ( $\mathrm{n}=7-8)$.

(D) Representative gross and microscopic images of hearts in the indicated groups. 40x objective (scale bar = $50 \mu \mathrm{m}$ ). (E) Statistical results for the cardiomyocyte cross-sectional area $(\mathrm{n} \geq 100$ cells). Total RNA was isolated from left ventricular of mice in different groups, and mRNA levels of (F) ANP, (G) BNP, and $(\mathrm{H}) \beta$-MHC determined by RT-PCR assay ( $\mathrm{n}=5-6) .{ }^{*} P<0.05$ vs. Sham+Veh; ${ }^{\#} P<0.05$ vs. $\mathrm{TAC}+\mathrm{Veh}$.

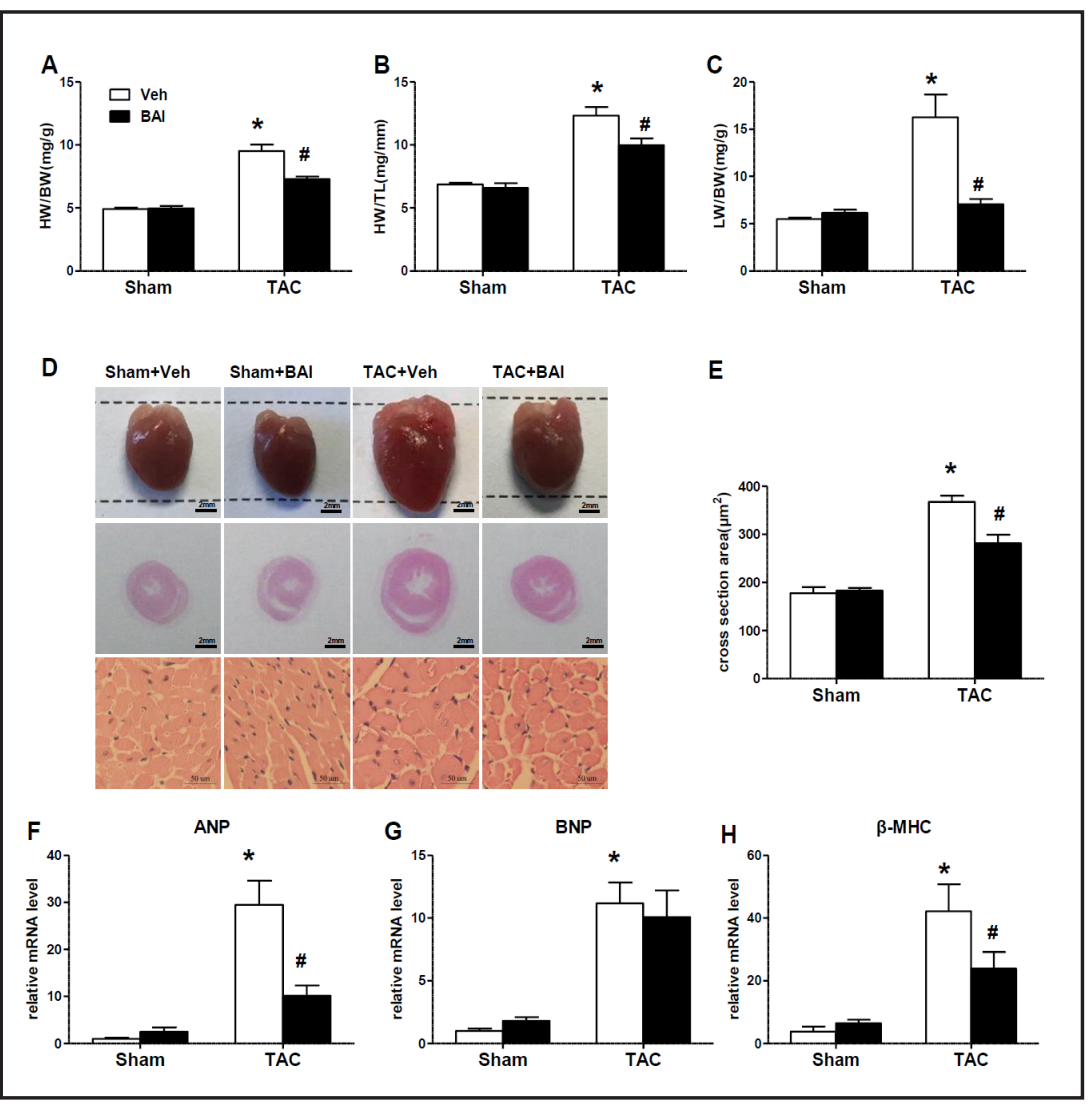

as measured by HW/BW ratio (Fig. 3A), HW/TL ratio (Fig. 3B), and cardiomyocyte crosssectional area (Fig. 3D and 3E). We also measured lung weight/body weight (LW/BW) ratio to further define the effect of baicalin on left ventricular dysfunction following TAC. As shown in Fig. 3C, the LW/BW ratio was elevated in the TAC+Veh group, and attenuated in the TAC+BAI groups (Fig. 3C). Furthermore, mRNA levels of hypertrophic hallmarks atrial natriuretic peptide (ANP) and myosin, heavy polypeptide 7 ( $\beta$-MHC) were shown to be induced by TAC and attenuated by baicalin treatment (Fig. $3 \mathrm{~F}$ and $3 \mathrm{H}$ ). However, we found no significant attenuation of natriuretic peptide type B (BNP) mRNA levels in TAC+BAI group compared with TAC+Veh group (Fig. 3G). Collectively, treatment of baicalin attenuates pressure overload-induced cardiac hypertrophy.

\section{Baicalin attenuates cardiac fibrosis after TAC surgery}

An important hallmark of pathological hypertrophy and heart failure is cardiac fibrosis [23], which is characterized by an increase in collagens and other extracellular matrix components in the interstitial and perivascular regions of the myocardium [4]. Therefore, we assessed cardiac fibrosis in the left ventricular myocardium following Masson trichrome staining. We found that both interstitial fibrosis and perivascular fibrosis were induced in TAC hearts and attenuated in baicalin-treated hearts (Fig. 4A). We further measured the collagen fractional area in the left ventricular myocardium, and obtained similar results (Fig. 4B). It is well known that the most abundant collagen types in the heart are type I and III, which account together for over $90 \%$ of the total collagen [23]. Previous studies have demonstrated that connective tissue growth factor (CTGF) is a major growth factor that promotes fibrosis in the hypertrophic heart $[23,24]$. Therefore, we detected mRNA levels of collagen I, collagen III and CTGF in these hearts under different stimuli. RT-PCR results demonstrated that pressure overload triggered elevation of collagen I, collagen III and CTGF mRNA levels in vehicle-treated hearts (Fig. 4C, 4D and 4E). In line with the Masson trichrome staining results, baicalin administration attenuated TAC-induced elevation of collagen I, 
Fig. 4. Baicalin attenuates cardiac fibrosis in vivo. (A) Masson trichrome staining of heart sections in the indicated groups. $40 \times$ objective (scale bar $=50 \mu \mathrm{m}$ ). (B) Quantification of the total collagen volume in (A) by measuring more than 30 fields. Relative mRNA levels of (C) Collagen I, (D) Collagen III and (E) CTGF in samples from different groups $(\mathrm{n}=5-6)$. ${ }^{*} P<0.05$ vs. Sham+Veh; ${ }^{\#} P<0.05$ vs. TAC+Veh.

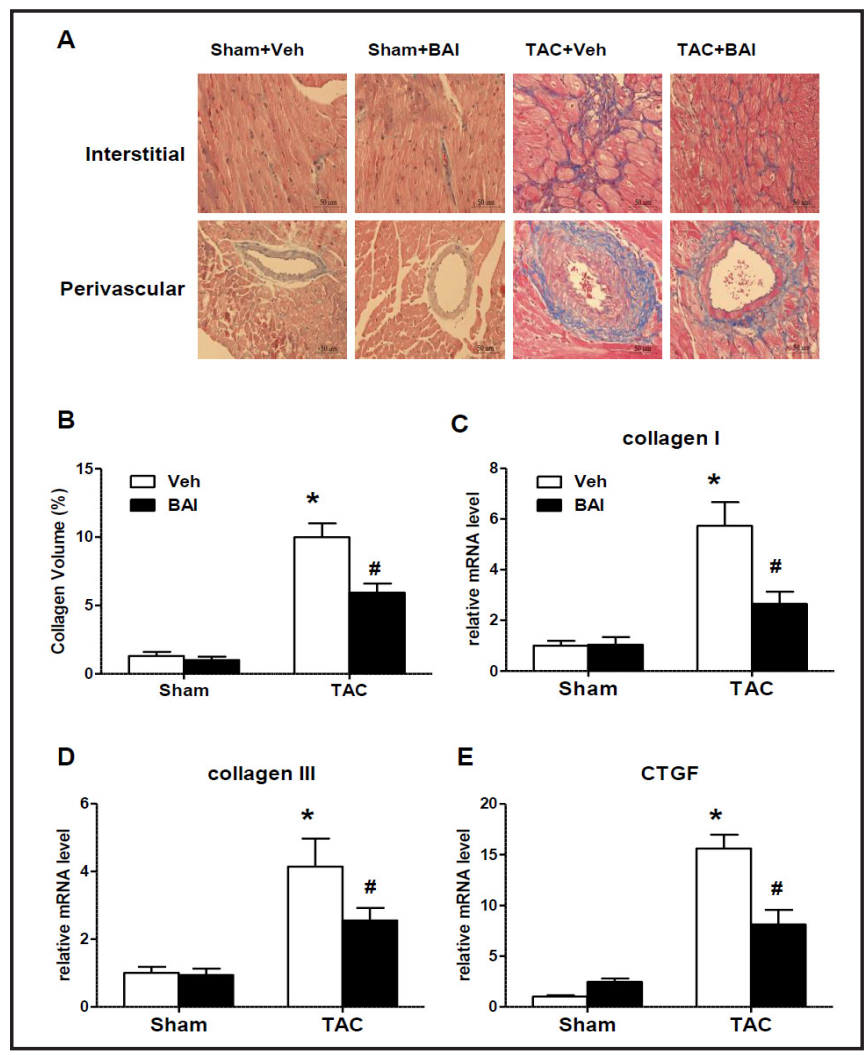

Fig. 5. Baicalin attenuates cardiac apoptosis in vivo. (A) TUNEL staining of heart sections in the indicated groups. $40 \times$ objective (scale bar $=50 \mu \mathrm{m}$ ). (B) The percentage of apoptosis cells was shown. ${ }^{*} P<0.05$ vs. Sham+Veh; ${ }^{\#} P<0.05$ vs. TAC+Veh. (C) The expressions of apoptosis-related proteins in heart tissue were detected by western blot. GAPDH was used as a loading control. (D) Bax/ $\mathrm{Bcl}-2$ ratio in the indicated groups were quantificated from (C) $(\mathrm{n}=4-6) .{ }^{*} P<0.05$ vs. Sham+Veh; ${ }^{\#} P<0.05$ vs. $\mathrm{TAC}+$ Veh.

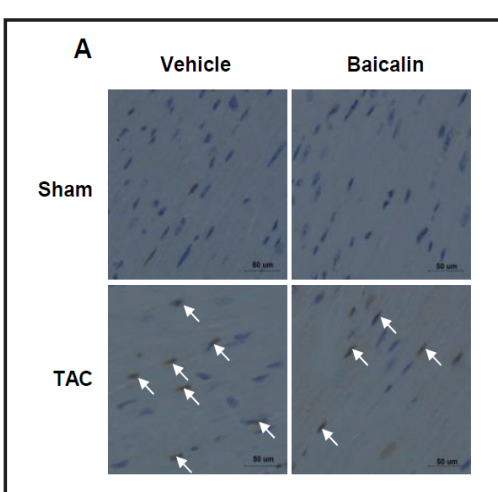

C

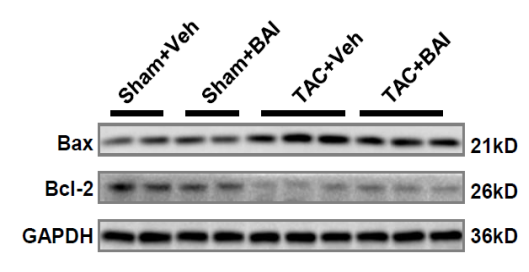

B

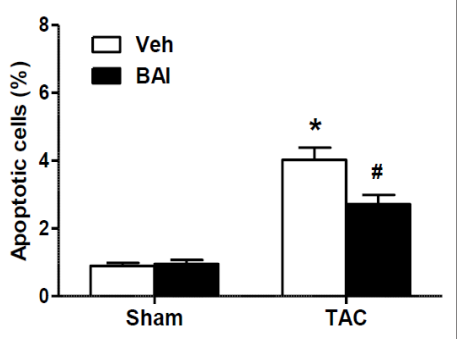

D

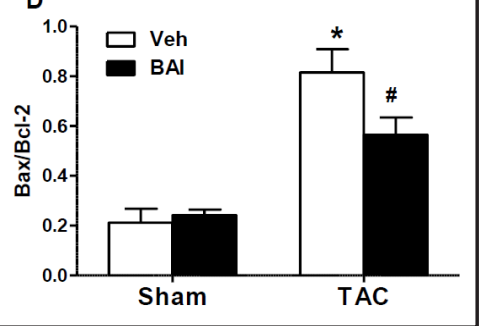

collagen III and CTGF mRNA levels (Fig. 4C, 4D and 4E). Collectively, these data indicated that oral administration of baicalin attenuates pressure overload-induced cardiac fibrosis.

\section{Baicalin reduces myocardial apoptosis following TAC surgery}

Previous studies have demonstrated that cardiomyocyte apoptosis is a characteristic cellular event and a causal mechanism in the progression of hypertrophy and heart failure $[25,26]$. Therefore, TUNEL staining was performed in order to investigate the effect of 


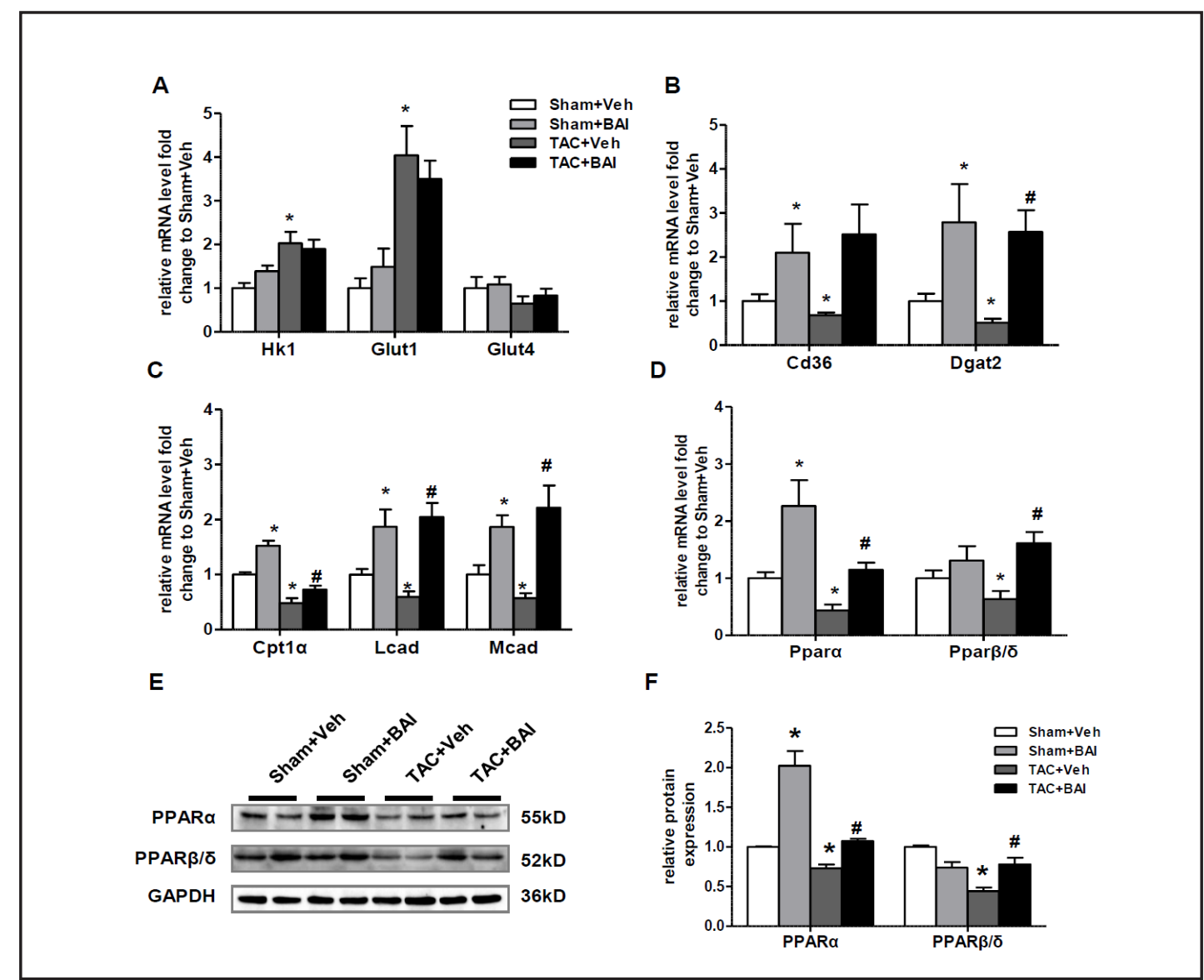

Fig. 6. Effects of baicalin on the expression of cardiac energy metabolic genes. (A, B and C) RT-PCR analysis of mRNA levels for glucose metabolism marker genes (A) and fatty acid metabolism marker genes (B and C) in mice hearts in the indicated groups. ${ }^{*} P<0.05$ vs. Sham $+V e h$; ${ }^{\#} P<0.05$ vs. TAC+Veh. ( $\mathrm{n}=5-6$ for A-C) (D) RT-PCR analysis of mRNA levels of PPAR $\alpha$ and PPAR $\beta / \delta$ in mice hearts in the indicated groups. ${ }^{*} P<0.05$ vs. Sham+Veh; ${ }^{\#} P<0.05$ vs. TAC+Veh. $(\mathrm{n}=5-6)(\mathrm{E})$ Protein levels of PPAR $\alpha$, PPAR $\beta / \delta$ and GAPDH in the indicated groups were shown. (F) Quantification of GAPDH-corrected protein levels of PPAR $\alpha$ and PPAR $\beta / \delta$, from (E). ${ }^{*} P<0.05$ vs. Sham+Veh; ${ }^{\#} P<0.05$ vs. TAC + Veh $(\mathrm{n}=4)$.

baicalin on cardiomyocyte apoptosis. We found that pressure overload lasting 4 weeks increased the number of TUNEL-positive nuclei (Fig. 5A and 5B). However, baicalin reduced TUNEL signals compared with the TAC+Veh group (Fig. 5A and $5 \mathrm{~B}$ ). To understand the mechanism by which baicalin inhibits cardiomyocyte apoptosis, we examined expression of the Bcl-2 family members through Western blot assay. The Bax/Bcl-2 ratio was remarkably increased in hearts of TAC+Veh group and decreased in hearts of TAC+BAI group (Fig. 5C and 5D). Baicalin alone had no effect on the Bax/Bcl-2 ratio. These results show that baicalin attenuates cardiomyocyte apoptosis, at least in part, through the regulation of pro-apoptotic Bax to anti-apoptotic Bcl-2 protein ratio.

Baicalin stimulates cardiac FAU genes expression without altering those involved in glucose utilization

Cardiac hypertrophy is associated with a decrease in FAU and an increase in glucose utilization [3]. To analyze the effect of baicalin treatment on energy metabolic genes expression, we performed RT-PCR to detect genes implicated in the transport and metabolism of glucose and fatty acids. We found that 4 weeks of pressure overload increased mRNA levels of glucose transporter member 1 (GLUT1) and hexokinase 1 (HK1) (Fig. 6A). However, treatment of baicalin did not influence their expression (Fig. 6A). Additionally, 


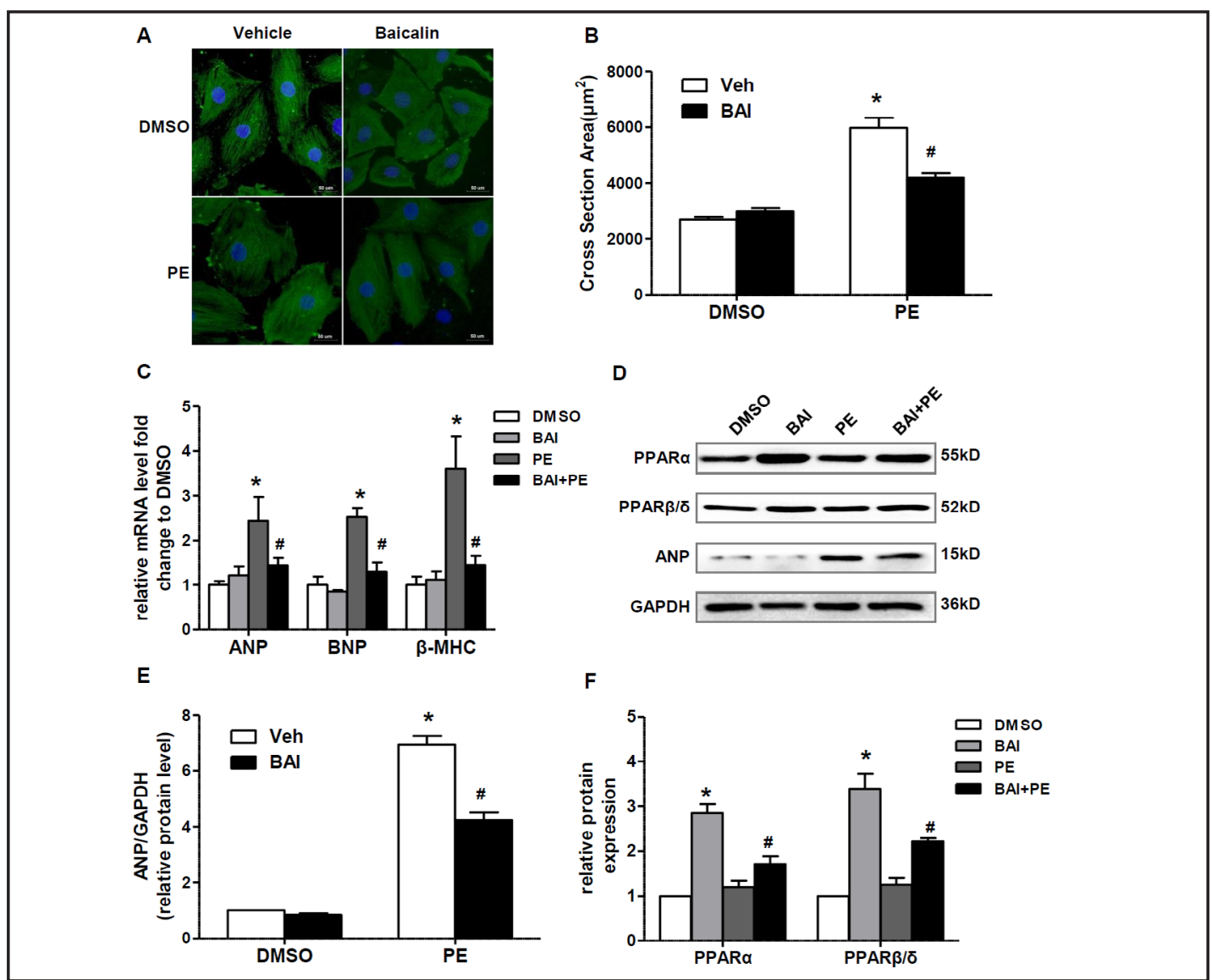

Fig. 7. Treatment with baicalin inhibits cardiac hypertrophy in vitro. (A) Representative fields of H9C2 cells stained with $\alpha$-actinin. Original magnification, $40 \times$ objective (scale bar $=50 \mu \mathrm{m}$ ). (B) Quantification of cell cross-sectional area from experiments shown in (A) by measuring more than 60 random cells. (C) mRNA levels of ANP, BNP, and $\beta$-MHC were analyzed by RT-PCR. (D) Protein levels of PPAR $\alpha$ PPAR $\beta / \delta$, ANP and GAPDH in the indicated groups were shown. (E and F) Quantification of GAPDH-corrected protein levels of ANP, PPAR $\alpha$ and PPAR $\beta / \delta$ from (D). These results were reproducible in 3 dependent experiments. ${ }^{*} P<0.05$ vs. DMSO; ${ }^{*} P<0.05$ vs. PE.

neither cardiac pressure overload nor baicalin treatment altered the expression of GLUT4 (Fig. 6A), which could translocate from intracellular stores to the cell surface through an insulin-dependent pathway [27]. Moreover, 4 weeks of cardiac pressure overload induced pronounced decrease in key genes involved in FAU (Fig. 6B and 6C). These genes included cluster of differentiation 36 (CD36), diacylglycerol 0-acyltransferase 2 (DGAT2), carnitine palmitoyltransferase $1 \alpha(\mathrm{CPT} 1 \alpha)$, long-chain acyl-coenzyme A dehydrogenase (LCAD), and medium-chain acyl-coenzyme A dehydrogenase (MCAD). Surprisingly, treatment of baicalin elevated mRNA levels of CD36, DGAT2, CPT1 $\alpha$, LCAD, and MCAD in both sham hearts and TAC hearts (Fig. 5B and 5C).

These findings suggest: (1) suppressed FAU genes expression and increased glucose utilization genes expression in hearts under 4 weeks of pressure overload; (2) increased FAU genes expression and unchanged glucose metabolism genes expression in hearts treated with baicalin in both sham and TAC hearts.

Baicalin improves cardiac energy metabolic rebalance through induction of PPAR $\alpha$ and $P P A R \beta / \delta$

PPARs regulate the expression of genes involved in glucose utilization, fatty acid metabolism and mitochondrial oxidative phosphorylation in the heart $[8,28]$. Therefore, we 

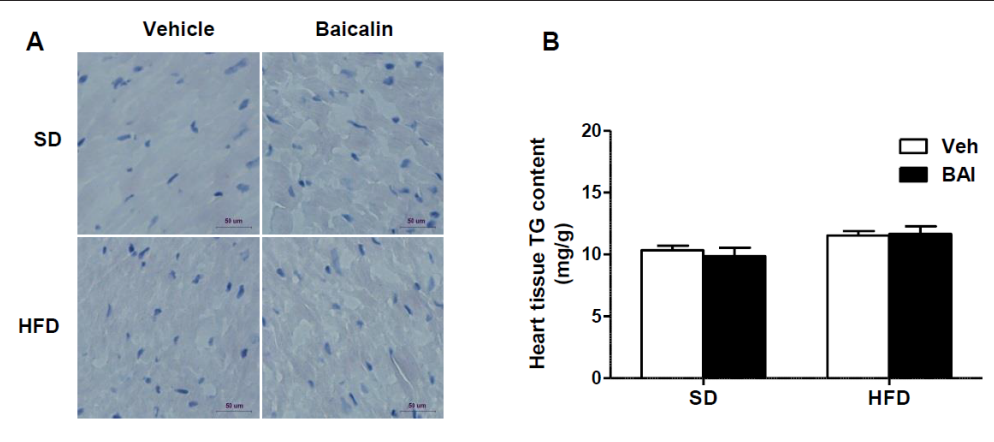

C

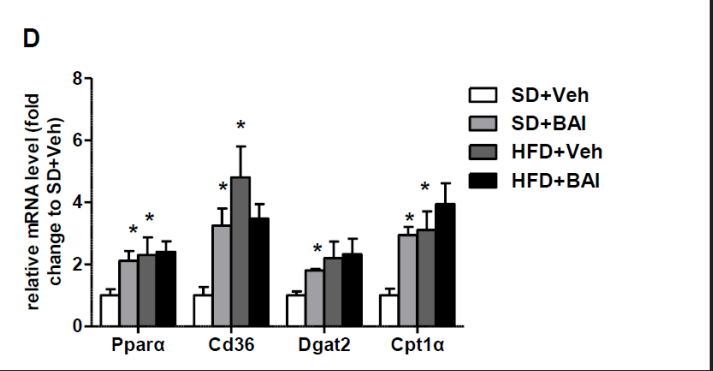

Fig. 8. Baicalin treatment presents no significant cardiac lipotoxicity. (A) Histologic appearance of ventricular tissue samples from mice after 4 weeks of HFD, accompanied with baicalin or vehicle treatment. (B and C) Myocardial TAG levels and serum NEFA levels were determined following HFD in mice in the indicated groups $(\mathrm{n}=5)$. ${ }^{*} P<0.05$ vs. Veh + SD. (D) mRNA levels of PPAR $\alpha$ CD36, DGAT2, and CPT1 $\alpha$ in the indicated groups were analyzed by RT-PCR. $(\mathrm{n}=5) .{ }^{*} P<0.05$ vs. Veh + SD.

performed RT-PCR to detect the myocardial expressions of PPAR $\alpha$ and PPAR $\beta / \delta$. We found decreased mRNA levels of PPAR $\alpha$ and PPAR $\beta / \delta$ in hearts under pressure overload (Fig. 6D). However, treatment of baicalin induced restoration in mRNA levels of these genes (Fig. 6D). Surprisingly, baicalin administration stimulated mRNA levels levels of PPAR $\alpha$ in Sham hearts (Fig. 6D). We further detected protein levels of PPAR $\alpha$ and PPAR $\beta / \delta$ by performing Western blot assay. Consistently, cardiac pressure over-load decreased protein levels of PPAR $\alpha$ and PPAR $\beta / \delta$ (Fig. 6E and 6F). Baicalin administration increased protein levels of PPAR $\alpha$ both in Sham and TAC hearts and restored PPAR $\beta / \delta$ protein level in hearts under hemodynamic stress (Fig. 6E and 6F). Collectively, these findings suggest decreased expression of PPAR $\alpha$ and PPAR $\beta / \delta$ in TAC hearts and restoration of these genes in baicalin-treated TAC hearts.

\section{Baicalin inhibits cardiomyocyte hypertrophy in vitro}

To detect the effect of baicalin on cardiomyocyte hypertrophy in vitro, H9C2 cells were cultured and treated with PE or DMSO in the presence or absence of baicalin. Cardiac hypertrophy can be monitored by increased myocyte cross-sectional area, and induction of fetal gene expression [29]. Cardiac myocytes were incubated with $50 \mu \mathrm{M}$ baicalin for 24 hours and subsequently treated with PE for 48 hours. There were no observable adverse effects by the treatment of DMSO or baicalin alone (data not shown). We found that 48 hours of PE induction resulted in marked increase in cardiac myocyte size, which was attenuated by treatment with baicalin (Fig. 7A and 7B). Meanwhile, PE-induced high expression of the hypertrophic hallmarks ANP, BNP and $\beta$-MHC were attenuated by treatment of baicalin (Fig. 7C). Further detection of ANP protein levels showed similar result (Fig. 7D and 7E). Furthermore, we detected increased protein levels of PPAR $\alpha$ and PPAR $\beta / \delta$ in cultured cardiomyocyte treated by baicalin (Fig. 7D and 7F). However, 48 hours of PE induction was not sufficient to influence protein levels of PPAR $\alpha$ and PPAR $\beta / \delta$ (Fig. 7D and 7F). Taken together, these data demonstrated that (1) baicalin attenuated PE-induced cardiac hypertrophy in vitro, and (2) baicalin increased protein levels of PPAR $\alpha$ and PPAR $\beta / \delta$ in cultured cardiomyocyte in the absence or presence of PE.

\section{KARGER}




\section{Cellular Physiology Cell Physiol Biochem 2017;41:849-864

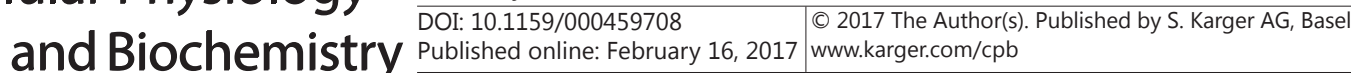 \\ Zhang et al.: Baicalin Attenuates Cardiac Hypertrophy}

Table 3. Parameters in adult mice fed 4 weeks of SD or HFD. Data are mean \pm SEM. ${ }^{\dagger} P<0.05$ vs. HFD + Veh. Abbreviations: SD, standard diet; HFD, high fat diet; BW, body weight

\begin{tabular}{lllll}
\hline Parameters & $\begin{array}{l}\text { SD + Veh } \\
(\mathrm{n}=5)\end{array}$ & $\begin{array}{l}\text { SD + BAI } \\
(\mathrm{n}=5)\end{array}$ & $\begin{array}{l}\text { HFD + Veh } \\
(\mathrm{n}=5)\end{array}$ & $\begin{array}{l}\text { HFD + BAI } \\
(\mathrm{n}=5)\end{array}$ \\
\hline BW before diet study (g) & $21.85 \pm 0.84$ & $21.98 \pm 0.66$ & $22.75 \pm 1.28$ & $22.14 \pm 1.02$ \\
BW 2 weeks after diet study (g) & $24.17 \pm 0.88$ & $23.84 \pm 0.56$ & $27.93 \pm 1.40$ & $25.56 \pm 1.32^{+}$ \\
BW 4 weeks after diet study (g) & $25.26 \pm 0.80$ & $24.85 \pm 1.03$ & $32.87 \pm 1.17$ & $29.10 \pm 1.72^{+}$ \\
Diet consumption (g/kg/day) & - & - & $3.58 \pm 0.20$ & $3.44 \pm 0.34$ \\
\hline
\end{tabular}

Long-term treatment of baicalin presents no significant lipotoxicity

MHC-PPAR $\alpha$ mice has been shown to develop cardiac dysfunction over time, and were prone to enhance myocardial lipid accumulation when treated with HFD [5, 8]. However, MHC-PPAR $\beta / \delta$ mice did not accumulate myocardial lipid and had normal cardiac function [8]. In the current study, the presence of increased PPAR $\alpha$ and its target genes expression and the absence of cardiac dysfunction phenotype in Sham mice treated with baicalin led us to explore whether baicalin alters myocardial lipid balance. Adult mice treated with baicalin or vehicle were fed HFD for 4 weeks to increase circulating nonesterified fatty acids (NEFA). Levels of myocardial neutral fat were visualized by oil red 0 staining, followed by extraction and quantification of triglyceride (TAG) contents. We found that hearts treated with baicalin did not accumulate abnormal levels of neutral lipid following 4 weeks of HFD (Fig. 8A). Consistently, quantification of TAG levels demonstrated no significant increase in myocardial TAG accumulation in baicalin-treated mice fed HFD compared with corresponding controls (Fig. 8B). While the food intake and serum NEFA levels were similar in mice treated with baicalin or vehicle in the HFD states (Table 3 and Fig. 8C), indicating that the supply of circulating NEFA was equivalent between different treatments. Moreover, we found that mice fed HFD displayed increased expression of FAU genes, and no further elevation when treated with baicalin (Fig. 8D). Taken together, these results demonstrate that Long-term treatment of baicalin presents no substantial lipotoxicity.

\section{Discussion}

Current therapeutic strategies toward heart failure emphasize inhibition of neurohumoral activation and normalization of hemodynamics [30]. Despite significant improvements in clinical diagnosis and efficiency of symptoms control, the progression of heart failure treatment remains unchanged with high rehospitalization rate. Novel therapeutic approaches are urgently needed [31]. Here, we provide an option of baicalin, which presents efficient attenuation of cardiac hypertrophy and mortality induced by pressure overload. Major findings of our study were as follows: (1) baicalin attenuated cardiac hypertrophy in response to hypertrophic stimuli both in vitro and in vivo; (2) baicalin-mediated cardioprotection was associated with suppressed cardiac hypertrophy, fibrosis, apoptosis and metabolic abnormality; and (3) baicalin-induced high expression of PPAR $\alpha$ presents no significant lipotoxicity.

Pressure overload is associated with numerous cardiac changes and responses including cardiac myocyte hypertrophy, fibrosis, apoptosis and metabolic abnormality [3, 32]. These features lead to cardiac dysfunction and pathological heart dilatation which progresses to heart failure. In the present study, cardiac hypertrophy was examined by assessing cardiac function during the time course of the study by echocardiography, measuring heart weight and cardiomyocyte cross-section area, and hypertrophic genes (ANP, BNP, and $\beta$-MHC) at study end. TAC-induced cardiac hypertrophy was attenuated by baicalin with a number of favourable outcomes including attenuation in cardiac dysfunction, smaller heart weight, lower myocyte cross-section area, and lower hypertrophic genes expression. 
To further explore potential mechanisms by which baicalin protect against pathological hypertrophy, we investigated the effect of baicalin on cardiac fibrosis and apoptosis. Cardiac fibrosis is a classical hallmark of pathological hypertrophy and is characterized by the expansion of collagens and other extracellular matrix components [23]. Previous research suggested that cardiac fibrosis not only causes systolic dysfunctions, but also disrupts the coordination of myocardial excitation-contraction coupling [4]. The present study demonstrated that baicalin attenuates cardiac fibrosis induced by pressure overload in vivo, as indicated by decreased extracellular matrix and lower expression of fibrogenic genes (Collagen I, Collagen III and CTGF). Additionally, apoptosis contributes to heart failure by decreasing the number of myocardial cells [33]. Even very low, but persistent, levels of apoptosis of myocardial cells gradually resulted in left ventricular dilation, systolic and diastolic dysfunction, and lethal dilated cardiomyopathy [26]. These studies show that a causal relationship exists between myocardial apoptosis and heart failure. Our results showed increased apoptosis signals in hearts under pressure overload. However, baicalin could inhibit the apoptosis by down-regulating the ratio of Bax/Bcl-2.

As the most avid ATP-consuming organ in the body, the heart possess quite low ATP reserve enough for only 10 contractions [34]. Therefore, the myocardium requires constant and robust mitochondrial ATP synthesis to maintain uninterrupted contraction and relaxation [30]. Previous studies demonstrated that abnormalities in cardiac lipid metabolism and energy production is a consistent feature of heart failure [30, 35]. Crucially, the progression of heart failure with pressure overload is associated with decreased PPAR $\alpha$ and PPAR $\beta / \delta$, and reactivation of PPAR $\alpha$, or PPAR $\beta / \delta$, is sufficient to reverse myocardial dysfunction and hypertrophy, respectively $[5,7,8,10]$. In the present study, we found myocardial dysfunction was ameliorated by baicalin treatment in TAC hearts, accompanied by reduced myocardial fibrosis, apoptosis signals, and profound restoration of PPAR $\alpha$ and PPAR $\beta / \delta$. These protective effects were also accompanied by unchanged expressions of glucose transport and utilization genes (GLUT1, GLUT4, and HK1) and increased FAU genes (CD36, DGAT2, CPT1 $\alpha$, LCAD, and MCAD). Previous studies suggested that cardiac expression of genes involved in glucose transport and utilization is repressed in MHC-PPAR $\alpha$ mice and induced in MHC-PPAR $\beta / \delta$ mice $[5,8]$. Additionally, PPAR $\alpha$ and PPAR $\beta / \delta$ exerted opposite transcriptional control of the GLUT4 promoter [8], which may explain the observed results including unchanged GLUT4 mRNA levels and increased both PPAR $\alpha$ and PPAR $\beta / \delta$ in baicalin-treated TAC heats. Besides, $\operatorname{PPAR} \alpha$ and PPAR $\beta / \delta$ share overlapping functions in regulating myocardial lipid metabolism [36]. Therefore, we cannot clearly conclude that baicalin mediated increase of FAU genes was fulfilled by induction of PPAR $\alpha$, or PPAR $\beta / \delta$.

Baicalin has been attracting growing interest from pharmaceuticalandfoodindustries due to its promising therapeutic uses [37]. In particular, this flavonoid has shown cardioprotective effects on variant disease models in vivo or in vitro. In cultured cardiomyocytes, ER stressinduced apoptosis were attenuated by baicalin via CHOP/eNOS/NO pathway [17]. In an acute myocardial infarction rats model, baicalin presented cardioprotective effests by modulating p38 MAPK cascades $[16,38]$. Additionally, baicalin may attenuate chronic hypoxia-induced pulmonary hypertension and cor pulmonale by downregulating the p38 MAPK/MMP-9 pathway [39]. Besides, evidence showed that baicalin attenuated HFD-induced obesity and

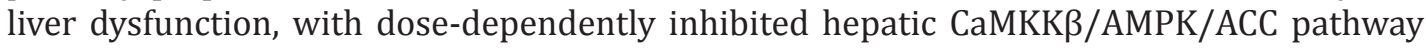
[40]. In the present study, we found that baicalin attenuated cardiac hypertrophy in response to hypertrophic stimuli, accompanied by restoration of PPAR $\alpha$ and PPAR $\beta / \delta$. Previously, reactivation of PPAR $\alpha$ with different ligands exhibited opposite effects on left ventricular hypertrophy in spontaneously hypertensive rat [7]. However, the protective effects of cardiac $\mathrm{PPAR} \beta / \delta$ restoration on cardiac hypertrophy were clearly demonstrated in vitro and in vivo $[10,12]$. Therefore, it is sufficient to concluded that the protective effects of baicalin were mediated through, at least in part, restoring cardiac PPAR $\beta / \delta$.

MHC-PPAR $\alpha$ mice exhibited signatures of cardiomyopathy including ventricular hypertrophy, enhanced expression of fetal genes, and systolic ventricular dysfunction $[5,8]$. Moreover, MHC-PPAR $\alpha$ mice exerted at least 15-fold increase in PPAR $\alpha$ level, and

\section{KARGER}




\section{Cellular Physiology Cell Physiol Biochem 2017;41:849-864

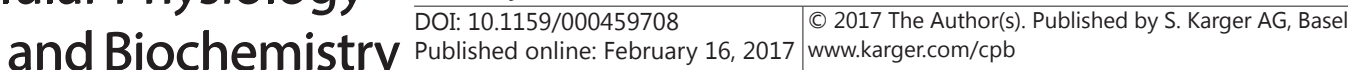 \\ Zhang et al.: Baicalin Attenuates Cardiac Hypertrophy}

enhanced myocardial lipid accumulation followed by HFD, which is possibly because of TAG synthesis beyond the capacity for oxidation [5, 8]. Therefore, we performed diet studies to further assess whether long-term administration of baicalin accelerate myocardial lipid accumulation. Mice were fed HFD for 4 weeks to increase circulating NEFA, whose localization at the luminal surface of coronary blood vessels may serve to activate PPAR $\alpha$ in heart tissue [41]. Activated PPAR $\alpha$ promotes myocardial fatty acid uptake (CD36), TAG synthesis (DGAT2), and fatty acid oxidation (CPT1 $\alpha$, LCAD, and MCAD). Here we detected no significant increase in myocardial TAG accumulation in baicalin-treated mice given HFD compared with corresponding controls. Perhaps baicalin-induced PPAR $\alpha$ is not as strong as cardiac-restricted overexpression of PPAR $\alpha$, and is not sufficient to induce severe myocardial neutral lipid accumulation. Therefore, oral administration of baicalin presents no significant myocardial lipotoxicity.

Taken together, these data suggest that oral administration of baicalin attenuated pressure overload induced cardiac dysfunction, high mortality and ventricular remodeling. These protective effects of baicalin treatment appear to be mediated by suppression of cardiac hypertrophy, fibrosis and apoptosis, and restoration of PPAR $\beta / \delta$.

\section{Acknowledgments}

We thank veterinarian Shunchang Zhou for animal study technical guidance. This study was supported by Hubei Science Foundation of China (No. 2012FF B 02508).

\section{Disclosure Statement}

The authors declare that they have no interests that could influence the publication of this paper.

\section{References}

1 Braunwald E: The war against heart failure: the Lancet lecture. The Lancet 2015;385:812-824.

2 Aronow BJ, Toyokawa T, Canning A, Haghighi K, Delling U, Kranias E, Molkentin JD, Dorn GW, 2nd: Divergent transcriptional responses to independent genetic causes of cardiac hypertrophy. Physiol Genomics 2001;6:19-28.

-3 Planavila A, Calvo RR, Vazquez-Carrera M: Peroxisome proliferator-activated receptors and the control of fatty acid oxidation in cardiac hypertrophy. Mini Rev Med Chem 2006;6:357-363.

-4 Berk BC, Fujiwara K, Lehoux S: ECM remodeling in hypertensive heart disease. J Clin Invest 2007;117:568575 .

5 Finck BN, Lehman JJ, Leone TC, Welch MJ, Bennett MJ, Kovacs A, Han X, Gross RW, Kozak R, Lopaschuk GD, Kelly DP: The cardiac phenotype induced by PPAR $\alpha$ overexpression mimics that caused by diabetes mellitus. J Clin Invest 2002;109:121-130.

6 Iemitsu M, Shimojo N, Maeda S, Irukayama-Tomobe Y, Sakai S, Ohkubo T, Tanaka Y, Miyauchi T: The benefit of medium-chain triglyceride therapy on the cardiac function of SHRs is associated with a reversal of metabolic and signaling alterations. Am J Physiol Heart Circ Physiol 2008;295:H136-144.

7 Ismael S, Purushothaman S, Harikrishnan VS, Nair RR: Ligand specific variation in cardiac response to stimulation of peroxisome proliferator-activated receptor-alpha in spontaneously hypertensive rat. Mol Cell Biochem 2015;406:173-182.

8 Burkart EM, Sambandam N, Han X, Gross RW, Courtois M, Gierasch CM, Shoghi K, Welch MJ, Kelly DP: Nuclear receptors PPARbeta/delta and PPARalpha direct distinct metabolic regulatory programs in the mouse heart. J Clin Invest 2007;117:3930-3939. 


\section{Cellular Physiology Cell Physiol Biochem 2017;41:849-864

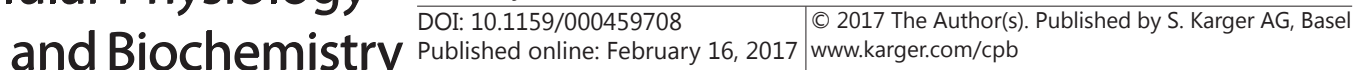 \\ Zhang et al.: Baicalin Attenuates Cardiac Hypertrophy}

99 Gilde AJ: Peroxisome Proliferator-Activated Receptor (PPAR) alpha and PPARbeta/delta, but not PPARgamma, Modulate the Expression of Genes Involved in Cardiac Lipid Metabolism. Circ Res 2003;92:518-524.

10 el Azzouzi H, Leptidis S, Dirkx E, Hoeks J, van Bree B, Brand K, McClellan EA, Poels E, Sluimer JC, van den Hoogenhof MM, Armand AS, Yin X, Langley S, Bourajjaj M, Olieslagers S, Krishnan J, Vooijs M, Kurihara H, Stubbs A, Pinto YM, Krek W, Mayr M, da Costa Martins PA, Schrauwen P, De Windt LJ: The hypoxia-inducible microRNA cluster miR-199a approximately 214 targets myocardial PPARdelta and impairs mitochondrial fatty acid oxidation. Cell Metab 2013;18:341-354.

11 Cheng L, Ding G, Qin Q, Huang Y, Lewis W, He N, Evans RM, Schneider MD, Brako FA, Xiao Y, Chen YE, Yang Q: Cardiomyocyte-restricted peroxisome proliferator-activated receptor-delta deletion perturbs myocardial fatty acid oxidation and leads to cardiomyopathy. Nat Med 2004;10:1245-1250.

-12 Planavila A, Rodriguezcalvo R, Jove M, Michalik L, Wahli W, Laguna J, Vazquezcarrera M: Peroxisome proliferator-activated receptor?/? activation inhibits hypertrophy in neonatal rat cardiomyocytes. Cardiovasc Res 2005;65:832-841.

13 Wei Z-F, Wang X-Q, Peng X, Wang W, Zhao C-J, Zu Y-G, Fu Y-J: Fast and green extraction and separation of main bioactive flavonoids from Radix Scutellariae. Ind Crops Prod 2015;63:175-181.

14 Liao P, Liu L, Wang B, Li W, Fang X, Guan S: Baicalin and geniposide attenuate atherosclerosis involving lipids regulation and immunoregulation in ApoE-/- mice. Eur J Pharmacol 2014;740:488-495.

15 Liu L, Liao P, Wang B, Fang X, Li W, Guan S: Oral administration of baicalin and geniposide induces regression of atherosclerosis via inhibiting dendritic cells in ApoE-knockout mice. Int Immunopharmacol 2014;20:197-204.

16 Liu X, Gu J, Fan Y, Shi H, Jiang M: Baicalin attenuates acute myocardial infarction of rats via mediating the mitogen-activated protein kinase pathway. Biol Pharm Bull 2013;36:988-994.

17 Shen M, Wang L, Yang G, Gao L, Wang B, Guo X, Zeng C, Xu Y, Shen L, Cheng K, Xia Y, Li X, Wang H, Fan L, Wang X: Baicalin protects the cardiomyocytes from ER stress-induced apoptosis: inhibition of CHOP through induction of endothelial nitric oxide synthase. PLoS One 2014;9:e88389.

18 Liu P, Yan S, Chen M, Chen A, Yao D, Xu X, Cai X, Wang L, Huang X: Effects of baicalin on collagen Iota and collagen IotaIotaIota expression in pulmonary arteries of rats with hypoxic pulmonary hypertension. Int J Mol Med 2015;35:901-908.

19 Liu Y, Shen Y, Zhu J, Liu M, Li X, Chen Y, Kong X, Song G, Qian L: Cardiac-specific PID1 overexpression enhances pressure overload-induced cardiac hypertrophy in mice. Cell Physiol Biochem 2015;35:19751985.

20 Alesutan I, Voelkl J, Stockigt F, Mia S, Feger M, Primessnig U, Sopjani M, Munoz C, Borst O, Gawaz M, Pieske B, Metzler B, Heinzel F, Schrickel JW, Lang F: AMP-activated protein kinase alpha1 regulates cardiac gap junction protein connexin 43 and electrical remodeling following pressure overload. Cell Physiol Biochem 2015;35:406-418.

21 Zhang Y, Wang C, Tian Y, Zhang F, Xu W, Li X, Shu Z, Wang Y, Huang K, Huang D: Inhibition of Poly(ADP-Ribose) Polymerase-1 Protects Chronic Alcoholic Liver Injury. Am J Pathol DOI:10.1016/j. ajpath.2016.08.016.

-22 Arany Z, Novikov M, Chin S, Ma Y, Rosenzweig A, Spiegelman BM: Transverse aortic constriction leads to accelerated heart failure in mice lacking PPAR-gamma coactivator 1alpha. Proc Natl Acad Sci U S A 2006;103:10086-10091.

-23 Creemers EE, Pinto YM: Molecular mechanisms that control interstitial fibrosis in the pressure-overloaded heart. Cardiovasc Res 2011;89:265-272.

24 Weber KT, Sun Y, Bhattacharya SK, Ahokas RA, Gerling IC: Myofibroblast-mediated mechanisms of pathological remodelling of the heart. Nat Rev Cardiol 2012;10:15-26.

25 Olivetti G, Abbi R, Quaini F, Kajstura J, Cheng W, Nitahara JA, Quaini E, Di Loreto C, Beltrami CA, Krajewski S, Reed JC, Anversa P: Apoptosis in the failing human heart. N Engl J Med 1997;336:1131-1141.

26 Wencker D, Chandra M, Nguyen K, Miao W, Garantziotis S, Factor SM, Shirani J, Armstrong RC, Kitsis RN: A mechanistic role for cardiac myocyte apoptosis in heart failure. J Clin Invest 2003;111:1497-1504.

27 Montessuit C, Lerch R: Regulation and dysregulation of glucose transport in cardiomyocytes. Biochim Biophys Acta 2013;1833:848-856. 


\section{Cellular Physiology Cell Physiol Biochem 2017;41:849-864

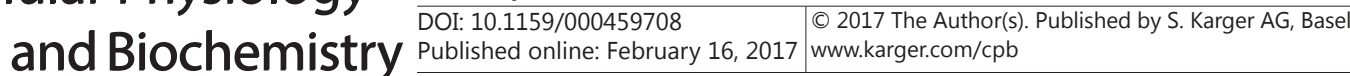 \\ Zhang et al.: Baicalin Attenuates Cardiac Hypertrophy}

28 Ventura-Clapier R, Garnier A, Veksler V: Transcriptional control of mitochondrial biogenesis: the central role of PGC-1alpha. Cardiovasc Res 2008;79:208-217.

29 Dorn GW, 2nd, Force T: Protein kinase cascades in the regulation of cardiac hypertrophy. J Clin Invest 2005;115:527-537.

30 Wang ZV, Li DL, Hill JA: Heart failure and loss of metabolic control. J Cardiovasc Pharmacol 2014;63:302313.

-31 Siddiqi N, Singh S, Beadle R, Dawson D, Frenneaux M: Cardiac metabolism in hypertrophy and heart failure: implications for therapy. Heart Fail Rev 2013;18:595-606.

-32 Tham YK, Bernardo BC, Ooi JY, Weeks KL, McMullen JR: Pathophysiology of cardiac hypertrophy and heart failure: signaling pathways and novel therapeutic targets. Arch Toxicol 2015;89:1401-1438.

33 Garg S, Narula J, Chandrashekhar Y: Apoptosis and heart failure: clinical relevance and therapeutic target. J Mol Cell Cardiol 2005;38:73-79.

34 Stanley WC, Recchia FA, Lopaschuk GD: Myocardial substrate metabolism in the normal and failing heart. Physiol Rev 2005;85:1093-1129.

-35 Razeghi P, Young ME, Alcorn JL, Moravec CS, Frazier OH, Taegtmeyer H: Metabolic gene expression in fetal and failing human heart. Circulation 2001;104:2923-2931.

-36 Yang Q Li Y: Roles of PPARs on regulating myocardial energy and lipid homeostasis. J Mol Med (Berl) 2007;85:697-706.

37 de Oliveira MR, Nabavi SF, Habtemariam S, Erdogan Orhan I, Daglia M, Nabavi SM: The effects of baicalein and baicalin on mitochondrial function and dynamics: A review. Pharmacol Res 2015;100:296-308.

-38 Sun SJ, Wu XP, Song HL, Li GQ: Baicalin ameliorates isoproterenol-induced acute myocardial infarction through iNOS, inflammation, oxidative stress and P38MAPK pathway in rat. Int J Clin Exp Med 2015;8:22063-22072.

-39 Yan S, Wang Y, Liu P, Chen A, Chen M, Yao D, Xu X, Wang L, Huang X: Baicalin Attenuates Hypoxia-Induced Pulmonary Arterial Hypertension to Improve Hypoxic Cor Pulmonale by Reducing the Activity of the p38 MAPK Signaling Pathway and MMP-9. Evid Based Complement Alternat Med 2016;2016:2546402.

40 Xi Y, Wu M, Li H, Dong S, Luo E, Gu M, Shen X, Jiang Y, Liu Y, Liu H: Baicalin Attenuates High Fat DietInduced Obesity and Liver Dysfunction: Dose-Response and Potential Role of CaMKKbeta/AMPK/ACC Pathway. Cell Physiol Biochem 2015;35:2349-2359.

-41 Yu YH, Ginsberg HN: Adipocyte signaling and lipid homeostasis: sequelae of insulin-resistant adipose tissue. Circ Res 2005;96:1042-1052. 